\title{
A Novel Resistance Measurement Method Based on Double Frequency Excitation Signals Eliminating the Infection of Distributed Capacitance
}

\author{
Chong-quan Zhong and Liang Zhao*, \\ Faculty of Electronic Information and Electrical Engineering \\ Dalian University of Technology \\ Dalian 116024, China \\ E-mail: zliangdut@qq.com
}

\begin{abstract}
The paper proposes a changing frequency method which is able to measure the value of the resistance. First, two AC square waves that have appropriate frequency are imposed on the partial circuit which is made up of electrode; and then, the critically rectified DC voltage from the partial circuit is measured; and next, the time constant of the partial circuit is calculated by solving the equation set; at last, the value of the resistance is obtained. The method eliminates the infection of the capacitance effectively, which will affect the metrical precision of the resistance value. The connection expression between the output DC voltage and the resistance to be measured and the capacitance is derived in the paper. And the infection of AC square wave amplitude to the metrical precision is eliminated effectively with the proportion method. The experimental results indicate that the metrical precision can arrive within $1 \%$ in such a condition: the partial resistance is $100 \mathrm{k} \Omega$, the capacitance is within the range of $500 \mathrm{pF} \sim 8000 \mathrm{pF}$, and the resistance to be measured is between $1 \mathrm{k} \Omega$ and $1 \mathrm{M} \Omega$. The experimental results show that this variable frequency AC excitation decoupled measurement method effectively eliminates the mutual influence between resistance and capacitance measurement of measuring object. Meanwhile, the paper also proposes the use of proportional method for elimination of the impact of AC square wave amplitude on measurement results, thereby effectively improving the measurement accuracy.
\end{abstract}

Keywords: Resistance measurement, Capacitance measurement, Resistancecapacitance decoupling, Soft sensing

\section{Introduction}

Conductivity is an important physical parameter of solid, liquid and organism, which has been widely used in chemical industry, metallurgy, biology, medicine, food and water and other production and scientific research departments [1-4]. According to the relative position detection element and the measured solution conductivity measurement is divided into non-contact measurement and contact measurement in two categories. Noncontact conductivity measurement using electromagnetic induction principle, also known as electromagnetic conductivity measurement, since the detection elements are not in direct contact with the test solution, used to measure the conductivity of acid, alkali and other corrosive liquids. Contactless conductivity measurement principle based on the electrolytic conductivity, the electrode is inserted into the test solution for the measurement, also known electrode conductivity measurement, is the most commonly used method for measuring the electrical conductivity. The resistance measurement method using the indirect method to measure the conductivity, the conductivity cell performance of a complex electrochemical system in the measurement process, the 
measurement result is mainly affected by polarization effects, capacitive effects and temperature in three areas [5-6].

Polarization Effect electrode lead by, when the distributed capacitance in the highfrequency excitation, long lead time effects are more serious, affecting the accuracy of the measurement results [7-8]. To eliminate this effect, must be measured using AC excitation source [9-10]. On the other hand, the effect of temperature variation on the conductivity measurement can be overcome by the temperature compensation. Temperature compensation in many ways, such as thermostats law, manual and automatic compensation Compensation Act law. Therefore, the capacitance effect becomes a major factor affecting the conductivity measurement. In order to overcome the effects of capacitance effects, people put forward a variety of measurement methods.

In 1970, Johnson DE and put forward a double-pulse method to measure conductivity [11-12], consider the effect of the electric double layer capacitor electrode leads and distributed capacitance obtained satisfactory. Due to ensure strict time synchronization, high hardware requirements to achieve them. Analog switches used to have a good switching characteristics and high switching speed, the selection of components for demanding, cost a lot of money. In paper [13], the researchers proposed an improved bridge, utilizing the principle of the oscillator, the conductivity obtained is converted to the resistance value of the known arm and a function of the amplitude of the excitation source. Using three test solution, the error is within 3\%. The method reduces the complexity of the manual operation, but low precision. [14] uses a phase-sensitive detection method to measure the conductivity of the solution, the accuracy can be controlled within $1 \%$. But there are shortcomings inherent phase-sensitive detection method, namely through $\mathrm{C}$, the signal must be the absolute difference between the switching signal $90^{\circ}$ in order to be completely filtered out. Meanwhile, when measuring the conductivity of the solution, due to the electric double layer capacitor can not be ignored, resulting in a phase difference between the inherent response signal and the excitation signal, the DC component filtered reduced, resulting in increased measurement error. Literature [15] proposed a frequency measurement method of indirect measurements of the conductivity, the conductivity is converted to a square wave signal proportional to the frequency of the signal, which to some extent inhibited the polarization effect and capacitance effects, achieve high precision, long-distance transmission. However, due to the conversion only in a small frequency range (1 $10 \mathrm{KHz}$ ) to ensure good linearity, in order to ensure accuracy, requires a corresponding electrode constant electrode to measure the inconvenience.Document [16] and other incentives to study the use of pulsed current stratum corneum nonlinear impedance, [17] literature, etc. using a square wave signal 12 as an incentive to solve the problem quickly measure bioelectrical impedance spectroscopy. However, the high frequency square wave signal attenuation phenomenon, has been an important issue plagued this detection method. Since the amplitude of the square wave signal a higher harmonic component will with increasing harmonic number of fast decay, in response to the higher harmonics of the signal to noise ratio decreases, resulting in the accuracy of impedance measurements fall

This paper presents a method for decoupled measurement of variable frequency resistance-capacitance (RC). Two appropriate frequency AC square wave signal sources are selected to stimulate the measured object, and then the output AC signal is converted into DC voltage by rectification. Next, the time constant of measuring object is solved based on the relationship between the DC voltage and the measuring object, which is further used to solve the values of the resistance and capacitance. 


\section{Principle of Variable Frequency RC Decoupling Measurement}

\subsection{Affiliations}

Figure 1 demonstrations the principle of variable frequency resistance measurement. The measuring probe electrode capacitance and lead capacitance, as well as capacitance characteristics of test substance are equivalent to the parallel connection of a capacitor (collectively referred to as the distributed capacitance) on the test substance, affecting the measurement of resistance. The dashed box in Figure 1 is a common electrode model [18], where dielectric resistance $R_{x}$ is connected in parallel with the distributed capacitance $C_{p}$, and divides the voltage with resistance $R_{1}$. Excitation source is a square wave with an amplitude of $E$, and a duty cycle of $50 \%$, whose frequency is continuously tunable.

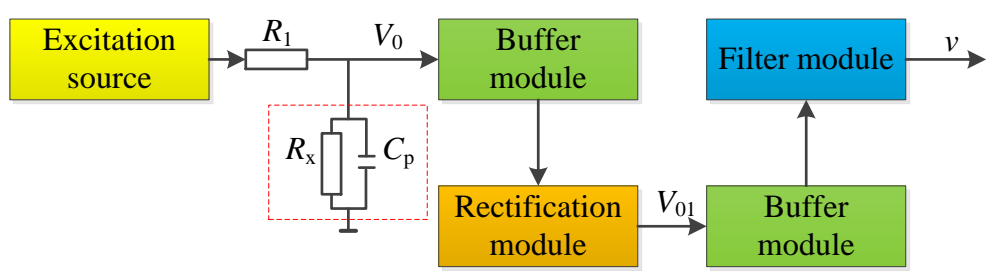

\section{Figure 1. Schematic of R-C Decoupling under AC Excitation}

Electrode output $V_{0}$ is buffered by operational amplifier, then goes through the precision half-wave rectifier to get voltage $V_{01}$, and then buffered again by operational amplifier, filter output to get DC value $v$. In Figure $1, R_{1}$ and electrode model (dashed box) constitute a RC network, and the charging and discharging time constant of this RC network is:

$$
\tau=\left(R_{1} \square R_{x}\right) C_{p}
$$

Figure 2 is charging and discharging waveform of the stimulating signal and output voltage, $V_{0}$. The dashed line is square wave excitation source with cycle as $2 T$, and the solid line is the output signal after entering into the steady state. As shown in Figure 2, $V_{1}(t)$ is the capacitor charging voltage, and $V_{2}(t)$ is the capacitor discharging voltage, according to the circuit theory, the expression of voltage $V_{0}$ in one cycle can be introduced as follows:

$$
\left\{\begin{array}{l}
V_{1}(t)=\frac{E R_{x}}{R_{1}+R_{x}}\left(1-e^{-\frac{t}{\tau}}\right)-\frac{E R_{x}}{R_{1}+R_{x}} \frac{1-e^{-\frac{T}{\tau}}}{1+e^{-\frac{T}{\tau}}} e^{-\frac{t}{\tau}} \\
V_{2}(t)=-\frac{E R_{x}}{R_{1}+R_{x}}\left(1-e^{-\frac{t-T}{\tau}}\right)-\frac{E R_{x}}{R_{1}+R_{x}} \frac{1-e^{-T / \tau}}{1+e^{-T / \tau}} e^{-\frac{t-T}{\tau}}
\end{array}\right.
$$




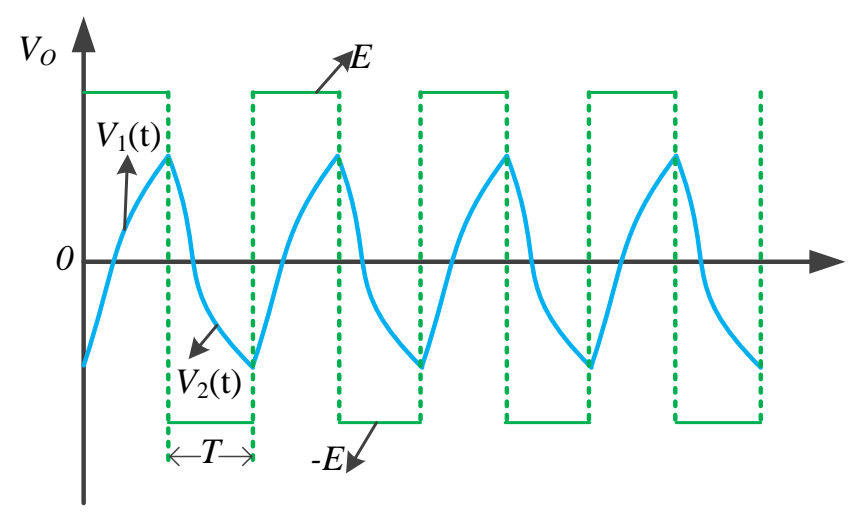

Figure 2. Wave of Object Voltage and Effecter

The rectified voltage waveform is shown in Figure $3, t_{A}, t_{B}$ are the zero crossing time, $V_{1}\left(t_{A}\right)=0, V_{2}\left(t_{B}\right)=0$, substituting them into equation (2.2) gives:

$$
\left\{\begin{array}{l}
t_{A}=\tau \ln \frac{2}{1+e^{-\frac{T}{\tau}}} \\
t_{B}=\tau \ln \frac{2}{1+e^{-\frac{T}{\tau}}}+T
\end{array}\right.
$$

By subsection integral within a 0 2T range, DC voltage is obtained:

$$
\begin{aligned}
& v=\frac{1}{2 T}\left(\int_{0}^{t_{A}}\left(-V_{1}(t)\right) d t+\int_{t_{B}}^{2 T}\left(-V_{2}(t)\right) d t\right) \\
& =\frac{E R_{X}}{R_{1}+R_{X}}\left(\frac{1}{2}+\frac{\tau}{T} \ln \frac{1+e^{-\frac{T}{\tau}}}{2}\right)
\end{aligned}
$$

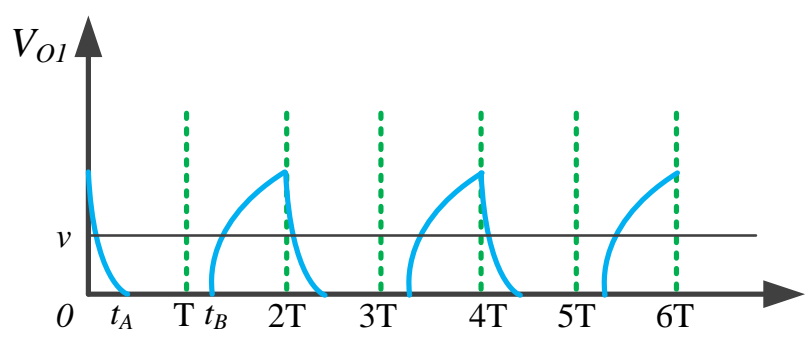

Figure 2. Half-wave Rectification Wave and Direct Voltage Output

It can be known from Equation 1 that $\tau$ is related to $R_{X}$ and $C_{P}$, which is an unknown value. Therefore, the DV value, $v$ is a binary function of $R_{X}$ and $\tau$, with the condition that the values of $E, R_{1}$ and $T$ are known. Setting the frequencies of two square waves as $f_{1}$ and $f_{2}$, and the corresponding pulse widths as: $T_{1}=\frac{1}{2 f_{1}}, T_{2}=\frac{1}{2 f_{2}}$. By substituting the values of $T_{1}$ and $T_{2}$ into the Equation 4, and obtain equation set as below: 


$$
\left\{\begin{array}{l}
v_{1}=\frac{E R_{X}}{R_{1}+R_{X}}\left(\frac{1}{2}+\frac{\tau}{T_{1}} \ln \frac{1+e^{-\frac{T_{1}}{\tau}}}{2}\right) \\
v_{2}=\frac{E R_{X}}{R_{1}+R_{X}}\left(\frac{1}{2}+\frac{\tau}{T_{2}} \ln \frac{1+e^{-\frac{T_{2}}{\tau}}}{2}\right)
\end{array}\right.
$$

Expression of $R_{X}$ can be derived from the expression of $v_{1}$ in Equation 5:

$$
R_{X}=\frac{\frac{v_{1}}{E} R_{1}}{\frac{1}{2}+\frac{\tau}{T_{1}} \ln \frac{1+e^{-\frac{T_{1}}{\tau}}}{2}-\frac{v_{1}}{E}}
$$

Furthermore, by dividing the two equations, and eliminating $R_{\mathrm{X}}$ yields:

$$
\tau\left(\frac{v_{1}}{v_{2}} \frac{1}{T_{2}} \ln \frac{1+e^{-\frac{T_{2}}{\tau}}}{2}-\frac{1}{T_{1}} \ln \frac{1+e^{-\frac{T_{1}}{\tau}}}{2}\right)-\frac{1}{2}\left(1-\frac{v_{1}}{v_{2}}\right)=0
$$

In this context, the time constant $\tau$ and measuring resistance $R_{X}$ can be determined according to the periods of excitation source $T_{1}, T_{2}$, measured rectified DC output voltages $v_{1}, v_{2}$ and the amplitude of excitation source $E$.

\subsection{Measurement Accuracy Analysis}

(1) Equation (7) is a single-variable nonlinear equation of $\tau$, the value of $\tau$ is obtained by Newton iteration method using matlab language programming, substituting $\tau$ value into equation (6), the value of measuring resistance $R_{X}$ can be got. Moreover, this value is independent of the distributed capacitance $C_{P}$, thus effectively eliminating the influence of distributed capacitance.

(2) Equation (7) is obtained by dividing two expressions in equation (5), which divides out the excitation source amplitude $E$. Measuring quantity $\tau$ is only related to the ratio of two DC voltages $v_{1}, v_{2}$ and excitation cycles $T_{1}, T_{2}$, which is independent of the amplitude of excitation source. Therefore, solving the time constant $\tau$ solved from equation (7) will not be affected by excitation source amplitude accuracy.

(3) Analyzing Equation (7), if two DC values are close, with a ratio approaching 1, then a relatively large calculation error will be resulted in during equation solving. So two appropriate frequencies must be selected, in order to ensure that the resulting two DC output voltages have a certain distinction.

(4) Analyzing formula (6), resistance of testing object $R_{X}$ is related to the excitation source amplitude, and if the amplitude of excitation source is known and highly precise, it can be obtained by calculation. However, circuit design must consider how to obtain a stable excitation source amplitude, where circuit overhead is relatively large. If a excitation source amplitude measurement method is adopted, and the amplitude of excitation source $E$ is measured using the measuring $v_{1}$ circuit, then the resistance $R_{X}$ DC value $v_{1}$ and the excitation source amplitude $E$ are in a comparative relationship, which can mutually offset the impacts of excitation source amplitude accuracy and measuring circuit, effectively improving the measurement accuracy. 


\section{Experimental Circuit Design}

\subsection{Square Wave Generating Circuit}

In order to prove the feasibility of the measurement method, the input excitation frequency and amplitude are required to have high accuracy and good stability. The input excitation source should be a $50 \%$ duty cycle square wave with continuously adjustable amplitude and frequency. The frequency accuracy of general signal generator could be satisfied the requirements of this experiment, but the accuracy and stability of amplitude are relatively poor. To this end, a precision steady excitation source amplitude circuit is designed to proceed the output amplitude of signal generator. The schematic is shown in Figure 4.

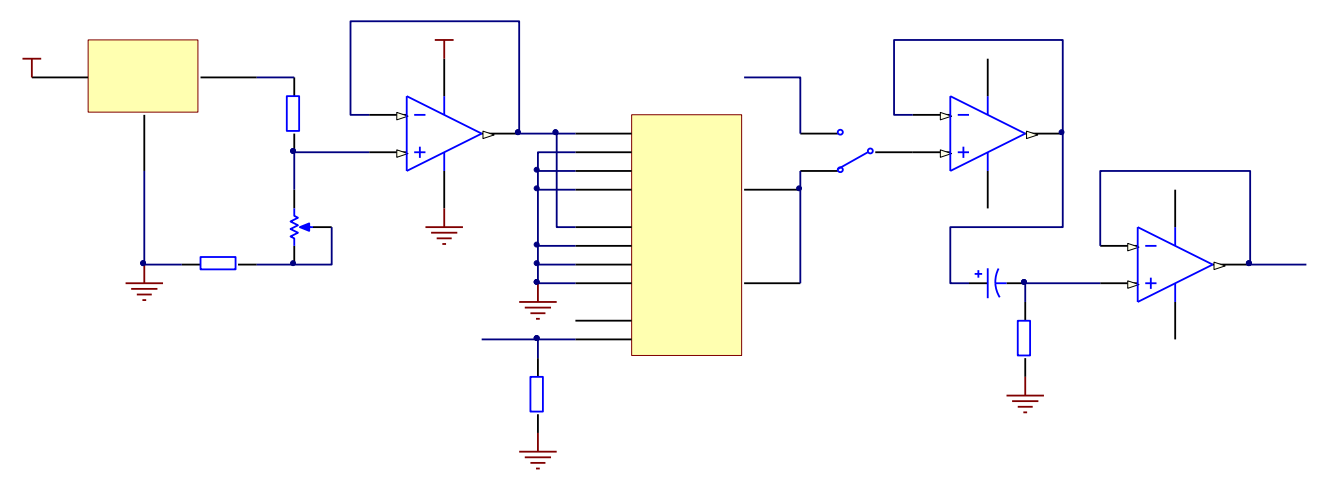

Figure 4. Effecter Generation Circuit

The integrated circuit MC1403, a high precision voltage reference source, is adopted here to generate the precise voltage $2.5 \mathrm{~V}$, and then divided via resistors and potentiometers to yield a stable $2 \mathrm{~V}$ voltage. The output voltage signal enter the 0 channel of analog switch CD4052 after buffering by operational amplifier TL084, while the other channels are grounded. Signal source is only required to have a stable frequency and be able to provide the high-low level needed by analog switch, greatly reducing the requirements on signal amplitude stability. With the addition of signal square wave, the output produces a square wave signal with very stable amplitude and frequency according to the frequency of the signal source.

The output signal of CD4052 is unipolar square wave, which can't be used directly to the measurement circuit. It should be passed through operational amplifier buffer and the high-pass filter constituted by capacitance and resistance to form a bipolar AC square wave with an amplitude of $\pm 1 \mathrm{~V}$, then buffered again through operational amplifier and connected to the input end of measuring circuit of testing object as the excitation source. Part B in Figure 4 shows the polarity changing process.

\subsection{RC Decoupling Circuit}

Figure 5 shows the experimental validation circuit of RC decoupling measurement. Divider resistance $R_{1}(100 \mathrm{~K} \Omega)$ is the precision resistance, the measuring resistance $R_{X}$ $(1 \mathrm{~K} \sim 10 \mathrm{M} \Omega)$ are all selected by high-precision metal film resistors, and distributed capacitance $C_{P}$ is simulated using ceramic capacitor nominally $500 \sim 8000 \mathrm{pF}$.

The output signal should be processed by four parts: firstly, is the buffered circuit consisted by the high input impedance operational amplifier LF356. Next, is the precision half-wave rectified circuit made up of by operational amplifier OP27, and thirdly, is a buffering circuit again by LF356. Finally, by using the RC low-pass filter to generate the DC signal. A switch $\mathrm{S}$ is added between the output of measuring object and the operational amplifier to switch between the measuring circuit output and the excitation 
source output, which is used to verify that the amplitude of excitation source has no effect on the measurement accuracy. In order to ensure the accuracy of the experimental measurement, six and a half digital multimeter HP34401A is used to measure the resistance and the output DC voltage.

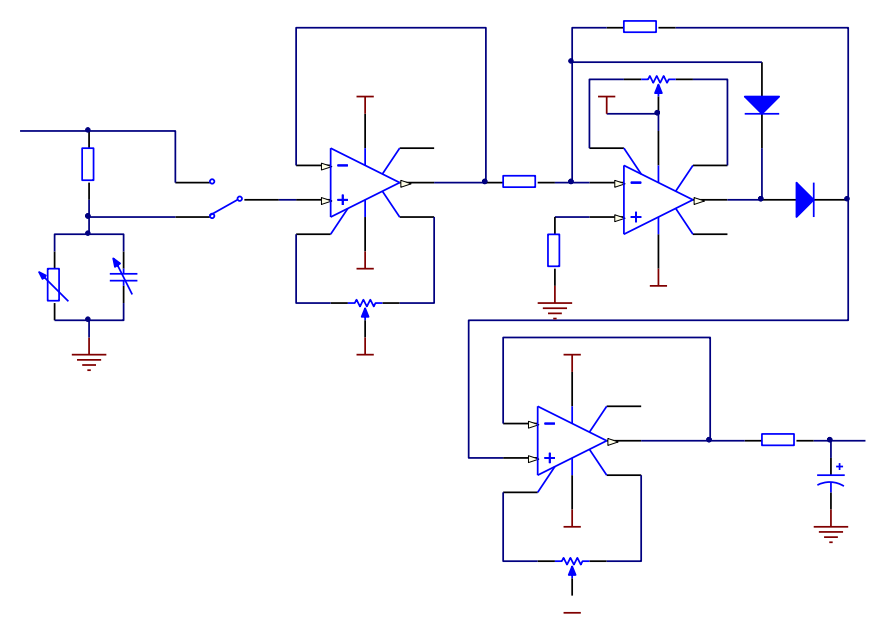

Figure 5. RC Decoupling Measurement Circuit

\section{Experimental Program and Testing Data}

\subsection{Resistance Measurement Experiment}

The switch $\mathrm{S}$ is set to A to measure the output signal. Distributed capacitance $C_{P}$ is taken as $500 \mathrm{pF}, 1000 \mathrm{pF}, 1500 \mathrm{pF}, 2000 \mathrm{pF}, 4000 \mathrm{pF}$ and $8000 \mathrm{pF}$, respectively, to conduct six groups of experiments, and the range of measuring object resistance $R_{X}$ is $1 \mathrm{~K} \sim 1 \mathrm{M} \Omega$ in each experiment. During the experiments, firstly, the values of $R_{X}, C_{P}, R_{1}, v_{1}$ and $v_{2}$ could be measured by the digital multimeter. Then, two frequencies $f_{1}, f_{2}$ are determined, and the corresponding pulse widths $T_{1}=1 / 2 f_{1}$ and $T_{2}=1 / 2 f_{2}$. Substituting these values into Equation 7 to calculate the time constant $\tau$. In addition, the resistance value of $R_{X 1}$ is calculated by the equation 6, and compared with the actual $R_{X}$, relative error is then obtained from the Equation 8:

$$
e=\frac{R_{X 1}-R_{X}}{R_{X}} 100 \%
$$

According to the time constant $\tau$ and solution resistance $R_{X 1}$, the distributed capacitance $C_{P 1}$ is calculated from the Equation 9.

$$
C_{P 1}=\frac{\tau}{R_{1} \square R_{X 1}}
$$

Tables 1 6 illustration the measurement results, and Figures 6 shows relative errors between measured and actual values of measured resistance under distributed capacitance $C_{P}$ of $500 \mathrm{pF}, 1000 \mathrm{pF}, 1500 \mathrm{pF}, 2000 \mathrm{pF}, 4000 \mathrm{pF}$ and $8000 \mathrm{pF}$, respectively. 
Table 1. Varies Resistance Verification Value under $C_{p}=500 p F$

\begin{tabular}{|l|l|l|l|l|c|c|c|c|}
\hline$R_{\mathrm{X}}(\Omega)$ & $f_{1}(\mathrm{~Hz})$ & $f_{2}(\mathrm{~Hz})$ & $v_{1}(\mathrm{mV})$ & $v_{2}(\mathrm{mV})$ & $T(\mathrm{us})$ & $R_{\mathrm{x} 1}(\Omega)$ & $e(\%)$ & $C_{\mathrm{p} 1}(\mathrm{pF})$ \\
\hline $1.0019 \mathrm{M}$ & 100 & 5300 & 448.98 & 198.14 & 46.913 & $1.0086 \mathrm{M}$ & 0.67 & 515.65 \\
\hline $764.85 \mathrm{~K}$ & 100 & 5400 & 436.82 & 194.09 & 45.607 & $768.27 \mathrm{~K}$ & 0.45 & 515.44 \\
\hline $470.62 \mathrm{~K}$ & 100 & 5800 & 407.79 & 180.76 & 42.548 & $472.46 \mathrm{~K}$ & 0.39 & 515.54 \\
\hline $399.80 \mathrm{~K}$ & 100 & 6000 & 397.57 & 174.94 & 41.236 & $400.73 \mathrm{~K}$ & 0.23 & 515.26 \\
\hline $301.36 \mathrm{~K}$ & 100 & 6400 & 371.51 & 163.98 & 38.723 & $301.74 \mathrm{~K}$ & 0.13 & 515.56 \\
\hline $239.56 \mathrm{~K}$ & 100 & 6800 & 349.35 & 154.38 & 36.356 & $239.92 \mathrm{~K}$ & 0.15 & 515.09 \\
\hline $200.62 \mathrm{~K}$ & 100 & 7200 & 330.76 & 146.43 & 34.289 & $201.09 \mathrm{~K}$ & 0.23 & 513.41 \\
\hline $99.778 \mathrm{~K}$ & 100 & 9700 & 248.05 & 108.45 & 25.749 & $99.867 \mathrm{~K}$ & 0.089 & 515.32 \\
\hline $81.842 \mathrm{~K}$ & 100 & $11 \mathrm{~K}$ & 223.66 & 96.128 & 23.202 & $81.89 \mathrm{~K}$ & 0.058 & 515.34 \\
\hline $51.146 \mathrm{~K}$ & 100 & 7000 & 168.47 & 112.965 & 17.576 & $51.192 \mathrm{~K}$ & 0.09 & 519.09 \\
\hline $29.930 \mathrm{~K}$ & 100 & $10 \mathrm{~K}$ & 114.84 & 77.676 & 12.022 & $29.946 \mathrm{~K}$ & 0.053 & 521.69 \\
\hline $22.122 \mathrm{~K}$ & 100 & $13 \mathrm{~K}$ & 90.341 & 60.328 & 9.6034 & $22.124 \mathrm{~K}$ & 0.0085 & 524.59 \\
\hline $15.006 \mathrm{~K}$ & 100 & $9 \mathrm{~K}$ & 65.111 & 53.9 & 6.9678 & $15.005 \mathrm{~K}$ & -0.0054 & 534.03 \\
\hline $9.9543 \mathrm{~K}$ & 100 & $13 \mathrm{~K}$ & 45.207 & 37.172 & 4.9657 & $9.9552 \mathrm{~K}$ & 0.009 & 548.46 \\
\hline $4.9967 \mathrm{~K}$ & 100 & $25 \mathrm{~K}$ & 23.712 & 18.938 & 2.9183 & $4.9827 \mathrm{~K}$ & -0.28 & 614.88 \\
\hline $2.9828 \mathrm{~K}$ & 100 & $21 \mathrm{~K}$ & 14.421 & 12.509 & 2.2867 & $2.9718 \mathrm{~K}$ & -0.37 & 792.33 \\
\hline 997.26 & 100 & $30 \mathrm{~K}$ & 4.873 & 8.841 & 2.5583 & 984.9 & -1.24 & 2623.1 \\
\hline
\end{tabular}

Table 2. Varies Resistance Verification Value under $C_{p}=100 p F$

\begin{tabular}{|c|l|l|l|l|l|l|l|l|}
\hline$R_{\mathrm{X}}(\Omega)$ & $f_{1}(\mathrm{~Hz})$ & $f_{2}(\mathrm{~Hz})$ & $v_{1}(\mathrm{mV})$ & $v_{2}(\mathrm{mV})$ & \multicolumn{1}{|c}{$T(\mathrm{us})$} & $R_{\mathrm{x} 1}(\Omega)$ & $e(\%)$ & $C_{\mathrm{p} 1}(\mathrm{pF})$ \\
\hline $1.0019 \mathrm{M}$ & 100 & 5400 & 443.01 & 110.09 & 91.785 & $1.008 \mathrm{M}$ & -0.11 & 1009.6 \\
\hline $764.85 \mathrm{~K}$ & 100 & 5600 & 431.26 & 106.17 & 89.343 & $765.26 \mathrm{~K}$ & 0.054 & 1010.2 \\
\hline $470.62 \mathrm{~K}$ & 100 & 6000 & 402.79 & 99.08 & 83.306 & $470.21 \mathrm{~K}$ & -0.087 & 1010.2 \\
\hline $399.80 \mathrm{~K}$ & 100 & 6200 & 391.03 & 95.84 & 80.874 & $400 \mathrm{~K}$ & 0.049 & 1010.9 \\
\hline $301.36 \mathrm{~K}$ & 100 & 6600 & 367.6 & 89.98 & 75.966 & $301.64 \mathrm{~K}$ & 0.091 & 1011.5 \\
\hline $239.56 \mathrm{~K}$ & 100 & 7000 & 345.81 & 84.78 & 71.402 & $239.66 \mathrm{~K}$ & 0.042 & 1012 \\
\hline $200.62 \mathrm{~K}$ & 100 & 7400 & 327.57 & 80.25 & 67.516 & $200.88 \mathrm{~K}$ & 0.13 & 1011.3 \\
\hline $99.778 \mathrm{~K}$ & 100 & 9900 & 246.29 & 59.91 & 50.594 & $99.835 \mathrm{~K}$ & 0.057 & 1012.7 \\
\hline $81.842 \mathrm{~K}$ & 100 & 5500 & 222.17 & 97.05 & 45.803 & $81.836 \mathrm{~K}$ & -0.067 & 1017.7 \\
\hline $51.146 \mathrm{~K}$ & 100 & 7300 & 167.64 & 72.87 & 34.59 & $51.178 \mathrm{~K}$ & 0.062 & 1021.8 \\
\hline $29.930 \mathrm{~K}$ & 100 & $11 \mathrm{k}$ & 114.45 & 48.54 & 23.611 & $29.939 \mathrm{~K}$ & 0.03 & 1024.8 \\
\hline $22.122 \mathrm{~K}$ & 100 & 6800 & 90.08 & 59.55 & 18.679 & $22.155 \mathrm{~K}$ & -0.033 & 1031.4 \\
\hline $15.006 \mathrm{~K}$ & 100 & 9400 & 64.981 & 42.85 & 13.544 & $15.002 \mathrm{k}$ & -0.025 & 1038.2 \\
\hline $9.9543 \mathrm{~K}$ & 100 & $14 \mathrm{k}$ & 45.142 & 29.07 & 9.5377 & $9.9533 \mathrm{~K}$ & 0.081 & 1053.6 \\
\hline $4.9967 \mathrm{~K}$ & 100 & $13 \mathrm{k}$ & 23.715 & 19.15 & 5.3801 & $4.987 \mathrm{~K}$ & -0.19 & 1132.6 \\
\hline $2.9828 \mathrm{~K}$ & 100 & $21 \mathrm{k}$ & 14.413 & 11.3 & 3.7326 & $2.9713 \mathrm{~K}$ & -0.38 & 1293.5 \\
\hline 997.26 & 100 & $31 \mathrm{k}$ & 4.873 & 3.59 & 3.0949 & 985.05 & -1.22 & 3172.8 \\
\hline
\end{tabular}


Table 3. Varies Resistance Verification Value under $C_{p}=1500 p F$

\begin{tabular}{|l|l|l|l|l|l|l|l|l|}
\hline$R_{\mathrm{X}}(\Omega)$ & $f_{1}(\mathrm{~Hz})$ & $f_{2}(\mathrm{~Hz})$ & $v_{1}(\mathrm{mV})$ & $v_{2}(\mathrm{mV})$ & $T(\mathrm{us})$ & $R_{\mathrm{x} 1}(\Omega)$ & $e(\%)$ & $C_{\mathrm{p} 1}(\mathrm{pF})$ \\
\hline $1.0019 \mathrm{M}$ & 100 & 3600 & 437.52 & 110.44 & 137.19 & $0.9999 \mathrm{M}$ & -0.2 & 1509.1 \\
\hline $764.85 \mathrm{~K}$ & 100 & 3700 & 425.58 & 107.6 & 133.17 & $760.53 \mathrm{~K}$ & -0.57 & 1506.8 \\
\hline $470.62 \mathrm{~K}$ & 100 & 4000 & 397.99 & 99.508 & 124.34 & $469.48 \mathrm{~K}$ & -0.24 & 1508.2 \\
\hline $399.80 \mathrm{~K}$ & 100 & 4100 & 386.42 & 96.999 & 120.65 & $398.97 \mathrm{~K}$ & -0.21 & 1508.9 \\
\hline $301.36 \mathrm{~K}$ & 100 & 4400 & 363.6 & 90.448 & 113.27 & $301.25 \mathrm{~K}$ & -0.04 & 1508.7 \\
\hline $239.56 \mathrm{~K}$ & 100 & 4700 & 342.23 & 84.734 & 106.35 & $239.28 \mathrm{~K}$ & -0.12 & 1508 \\
\hline $200.62 \mathrm{~K}$ & 100 & 4900 & 324.35 & 81.202 & 100.61 & $200.59 \mathrm{~K}$ & -0.017 & 1507.78 \\
\hline $99.778 \mathrm{~K}$ & 100 & 6600 & 244.49 & 60.382 & 75.218 & $99.758 \mathrm{~K}$ & -0.02 & 1506.2 \\
\hline $81.842 \mathrm{~K}$ & 100 & 7300 & 220.77 & 54.455 & 67.946 & $81.824 \mathrm{~K}$ & -0.022 & 1609.9 \\
\hline $51.146 \mathrm{~K}$ & 100 & 4800 & 166.83 & 74.208 & 51.344 & $51.167 \mathrm{~K}$ & -0.04 & 1616.9 \\
\hline $29.930 \mathrm{~K}$ & 100 & 7100 & 114.07 & 50.209 & 34.986 & $29.933 \mathrm{~K}$ & -0.011 & 1518.6 \\
\hline $22.122 \mathrm{~K}$ & 100 & 9100 & 89.865 & 39.159 & 27.587 & $22.177 \mathrm{~K}$ & -0.021 & 1523.2 \\
\hline $15.006 \mathrm{~K}$ & 100 & 6300 & 64.871 & 43.044 & 20.016 & $15.004 \mathrm{~K}$ & -0.012 & 1534.2 \\
\hline $9.9543 \mathrm{~K}$ & 100 & 9100 & 45.079 & 29.698 & 14.023 & $9.9517 \mathrm{~K}$ & -0.026 & 1549.3 \\
\hline $4.9967 \mathrm{~K}$ & 100 & 8600 & 23.695 & 19.359 & 7.7543 & $4.986 \mathrm{~K}$ & -0.21 & 1632.8 \\
\hline $2.9828 \mathrm{~K}$ & 100 & $14 \mathrm{~K}$ & 14.394 & 11.523 & 5.1756 & $2.9685 \mathrm{~K}$ & -0.43 & 1795.3 \\
\hline 997.26 & 100 & $21 \mathrm{~K}$ & 4.87 & 3.844 & 3.6396 & 984.58 & -1.27 & 3733 \\
\hline
\end{tabular}

Table 4. Varies Resistance Verification Value under $C_{p}=2000 p F$

\begin{tabular}{|l|l|l|l|l|l|l|l|l|}
\hline$R_{\mathrm{X}}(\Omega)$ & $f_{1}(\mathrm{~Hz})$ & $f_{2}(\mathrm{~Hz})$ & $v_{1}(\mathrm{mV})$ & $v_{2}(\mathrm{mV})$ & $T(\mathrm{us})$ & $R_{\mathrm{x} 1}(\Omega)$ & $e(\%)$ & $C_{\mathrm{p} 1}(\mathrm{pF})$ \\
\hline $1.0019 \mathrm{M}$ & 100 & 2800 & 431.83 & 108.18 & 180.4 & $1.0005 \mathrm{M}$ & -0.14 & 1984.3 \\
\hline $764.85 \mathrm{~K}$ & 100 & 2800 & 420.39 & 107.96 & 175.33 & $760.15 \mathrm{M}$ & -0.62 & 1984 \\
\hline $470.62 \mathrm{~K}$ & 100 & 3100 & 393.58 & 97.752 & 163.59 & $470 \mathrm{~K}$ & -0.13 & 1984 \\
\hline $399.80 \mathrm{~K}$ & 100 & 3200 & 382.19 & 94.718 & 158.6 & $398.85 \mathrm{~K}$ & -0.24 & 1983.7 \\
\hline $301.36 \mathrm{~K}$ & 100 & 3400 & 359.88 & 89.153 & 148.89 & $301.2 \mathrm{~K}$ & -0.05 & 1983.1 \\
\hline $239.56 \mathrm{~K}$ & 100 & 3600 & 338.93 & 84.207 & 139.78 & $239.2 \mathrm{~K}$ & -0.15 & 1982.1 \\
\hline $200.62 \mathrm{~K}$ & 100 & 3800 & 321.5 & 79.778 & 132.29 & $200.74 \mathrm{~K}$ & 0.057 & 1981.9 \\
\hline $99.778 \mathrm{~K}$ & 100 & 5000 & 242.83 & 60.513 & 99.05 & $99.75 \mathrm{~K}$ & -0.03 & 1983.5 \\
\hline $81.842 \mathrm{~K}$ & 100 & 5600 & 219.56 & 54.055 & 89.338 & $81.909 \mathrm{~K}$ & 0.08 & 1984.1 \\
\hline $51.146 \mathrm{~K}$ & 100 & 7400 & 166.07 & 40.804 & 67.297 & $51.161 \mathrm{~K}$ & 0.03 & 1988.4 \\
\hline $29.930 \mathrm{~K}$ & 100 & 5400 & 113.75 & 50.229 & 45.992 & $29.944 \mathrm{~K}$ & 0.05 & 1995.9 \\
\hline $22.122 \mathrm{~K}$ & 100 & 7000 & 89.687 & 38.861 & 36.247 & $22.129 \mathrm{~K}$ & 0.03 & 2000.4 \\
\hline $15.006 \mathrm{~K}$ & 100 & $10 \mathrm{~K}$ & 64.754 & 27.747 & 25.646 & $15 \mathrm{~K}$ & -0.4 & 1966.2 \\
\hline $9.9543 \mathrm{~K}$ & 100 & 7000 & 450.076 & 29.583 & 18.428 & $9.9644 \mathrm{~K}$ & 0.1 & 2033.6 \\
\hline $4.9967 \mathrm{~K}$ & 100 & $13 \mathrm{~K}$ & 23.707 & 15.434 & 10.043 & $4.992 \mathrm{~K}$ & -0.094 & 2112.4 \\
\hline $2.9828 \mathrm{~K}$ & 100 & $11 \mathrm{~K}$ & 14.43 & 11.522 & 6.6666 & $2.9774 \mathrm{~K}$ & -0.18 & 2305.7 \\
\hline 997.26 & 100 & $32 \mathrm{~K}$ & 4.878 & 3.15 & 4.136 & 986.35 & -1.09 & 4234.6 \\
\hline
\end{tabular}


Table 5. Varies Resistance Verification Value under $C_{p}=4000 p F$

\begin{tabular}{|l|l|l|l|l|l|l|l|l|}
\hline$R_{\mathrm{X}}(\Omega)$ & $f_{1}(\mathrm{~Hz})$ & $f_{2}(\mathrm{~Hz})$ & $v_{1}(\mathrm{mV})$ & $v_{2}(\mathrm{mV})$ & $T(\mathrm{us})$ & $R_{\mathrm{x} 1}(\Omega)$ & $e(\%)$ & $C_{\mathrm{p} 1}(\mathrm{pF})$ \\
\hline $1.0019 \mathrm{M}$ & 10 & 1300 & 450.28 & 112.24 & 373.71 & $1.011 \mathrm{M}$ & 0.91 & 4106.7 \\
\hline $764.85 \mathrm{~K}$ & 10 & 1400 & 437.65 & 104.72 & 362.62 & $763.48 \mathrm{~K}$ & -0.18 & 4101.1 \\
\hline $470.62 \mathrm{~K}$ & 10 & 1500 & 408.79 & 97.716 & 338.57 & $472.5 \mathrm{~K}$ & 0.4 & 4102.2 \\
\hline $399.80 \mathrm{~K}$ & 10 & 1500 & 396.3 & 97.46 & 328.21 & $399.7 \mathrm{~K}$ & -0.026 & 4103.2 \\
\hline $301.36 \mathrm{~K}$ & 10 & 1600 & 372.51 & 91.356 & 308.44 & $302.33 \mathrm{~K}$ & 0.32 & 4104.6 \\
\hline $239.56 \mathrm{~K}$ & 10 & 1700 & 349.94 & 86.029 & 289.3 & $239.61 \mathrm{~K}$ & 0.02 & 4101.3 \\
\hline $200.62 \mathrm{~K}$ & 10 & 1800 & 331.48 & 81.198 & 274.23 & $201.24 \mathrm{~K}$ & 0.31 & 4105 \\
\hline $99.778 \mathrm{~K}$ & 10 & 2400 & 248.32 & 60.94 & 204.83 & $99.791 \mathrm{~K}$ & 0.013 & 4100.9 \\
\hline $81.842 \mathrm{~K}$ & 10 & 2700 & 224.08 & 54.199 & 184.85 & $81.973 \mathrm{~K}$ & 0.16 & 4103.5 \\
\hline $51.146 \mathrm{~K}$ & 10 & 3600 & 168.7 & 40.649 & 139.02 & $51.219 \mathrm{~K}$ & 0.14 & 4104.3 \\
\hline $29.930 \mathrm{~K}$ & 10 & 5300 & 115.04 & 27.551 & $94.9=25$ & $29.986 \mathrm{~K}$ & 0.19 & 4114.9 \\
\hline $22.122 \mathrm{~K}$ & 10 & 6700 & 90.505 & 21.721 & 74.873 & $22.158 \mathrm{~K}$ & 0.16 & 4127.8 \\
\hline $15.006 \mathrm{~K}$ & 10 & 9300 & 65.207 & 15.531 & 54.353 & $150.23 \mathrm{~K}$ & 0.12 & 4161.4 \\
\hline $9.9543 \mathrm{~K}$ & 10 & 6700 & 45.294 & 19.458 & 37.846 & $9.9727 \mathrm{~K}$ & 0.18 & 4173.5 \\
\hline $4.9967 \mathrm{~K}$ & 10 & $13 \mathrm{~K}$ & 23.813 & 9.815 & 20.57 & $5.0038 \mathrm{~K}$ & 0.14 & 4316.6 \\
\hline $2.9828 \mathrm{~K}$ & 10 & $10 \mathrm{~K}$ & 14.454 & 9.454 & 12.816 & $2.9779 \mathrm{~K}$ & -0.16 & 4447.5 \\
\hline 997.26 & 10 & $15 \mathrm{~K}$ & 4.897 & 3.63 & 6.2685 & 989.26 & -0.8 & 6399.2 \\
\hline
\end{tabular}

Table 6. Varies Resistance Verification Value under $C_{p}=8000 p F$

\begin{tabular}{|l|l|l|l|l|c|c|c|c|}
\hline$R_{\mathrm{X}}(\Omega)$ & $f_{1}(\mathrm{~Hz})$ & $f_{2}(\mathrm{~Hz})$ & $v_{1}(\mathrm{mV})$ & $v_{2}(\mathrm{mV})$ & $T(\mathrm{us})$ & $R_{\mathrm{x} 1}(\Omega)$ & $e(\%)$ & $C_{\mathrm{p} 1}(\mathrm{pF})$ \\
\hline $1.0019 \mathrm{M}$ & 10 & 700 & 445.54 & 106.01 & 738.02 & $1.0074 \mathrm{M}$ & 0.54 & 8122.8 \\
\hline $764.85 \mathrm{~K}$ & 10 & 700 & 433.57 & 105.76 & 718.03 & $767.71 \mathrm{~K}$ & 0.37 & 8115.6 \\
\hline $470.62 \mathrm{~K}$ & 10 & 700 & 404.94 & 105.13 & 669.96 & $472.11 \mathrm{~K}$ & 0.32 & 8118.7 \\
\hline $399.80 \mathrm{~K}$ & 10 & 800 & 392.94 & 92.813 & 649.12 & $400.71 \mathrm{~K}$ & 0.23 & 8111.1 \\
\hline $301.36 \mathrm{~K}$ & 10 & 800 & 369.34 & 92.312 & 609.89 & $302.23 \mathrm{~K}$ & 0.29 & 8116.8 \\
\hline $239.56 \mathrm{~K}$ & 10 & 900 & 347.35 & 82.436 & 572.71 & $240.03 \mathrm{~K}$ & 0.2 & 8113.1 \\
\hline $200.62 \mathrm{~K}$ & 10 & 900 & 328.93 & 82.071 & 542.01 & $201.11 \mathrm{~K}$ & 0.24 & 8115.1 \\
\hline $99.778 \mathrm{~K}$ & 10 & 1200 & 247.09 & 61.597 & 405.2 & $99.918 \mathrm{~K}$ & 0.14 & 8107.3 \\
\hline $81.842 \mathrm{~K}$ & 10 & 1400 & 222.93 & 52.94 & 365.63 & $81.959 \mathrm{~K}$ & 0.14 & 8117.4 \\
\hline $51.146 \mathrm{~K}$ & 10 & 1800 & 168.04 & 41.083 & 274.81 & $51.208 \mathrm{~K}$ & 0.12 & 8114.7 \\
\hline $29.930 \mathrm{~K}$ & 10 & 2700 & 114.67 & 27.421 & 187.16 & $29.961 \mathrm{~K}$ & 0.1 & 8118.3 \\
\hline $22.122 \mathrm{~K}$ & 10 & 3400 & 90.258 & 21.742 & 147.27 & $22.138 \mathrm{~K}$ & 0.074 & 8125.2 \\
\hline $15.006 \mathrm{~K}$ & 10 & 4700 & 65.127 & 15.667 & 106.56 & $15.027 \mathrm{~K}$ & 0.14 & 8157.1 \\
\hline $9.9543 \mathrm{~K}$ & 10 & 3400 & 45.214 & 19.518 & 74.204 & $9.9644 \mathrm{~K}$ & 0.1 & 8188.9 \\
\hline $4.9967 \mathrm{~K}$ & 10 & 6500 & 23.759 & 10.082 & 39.646 & $4.9946 \mathrm{~K}$ & -0.04 & 8334.3 \\
\hline $2.9828 \mathrm{~K}$ & 10 & $11 \mathrm{k}$ & 14.452 & 5.797 & 25.171 & $2.9786 \mathrm{~K}$ & -0.14 & 8702.3 \\
\hline 997.26 & 10 & $15 \mathrm{~K}$ & 4.918 & 2.872 & 10.67 & 993.67 & -0.36 & 10845 \\
\hline
\end{tabular}

As can be seen from Figure 6, when the capacitance changes, resistance range can still maintain measurement accuracy remains within $1 \%$ between $1 \mathrm{~K} \sim 1 \mathrm{M} \Omega$. Besides, the measured value of capacitance has a higher accuracy when the resistance is larger; and has a slightly lower accuracy when the resistance is smaller. 


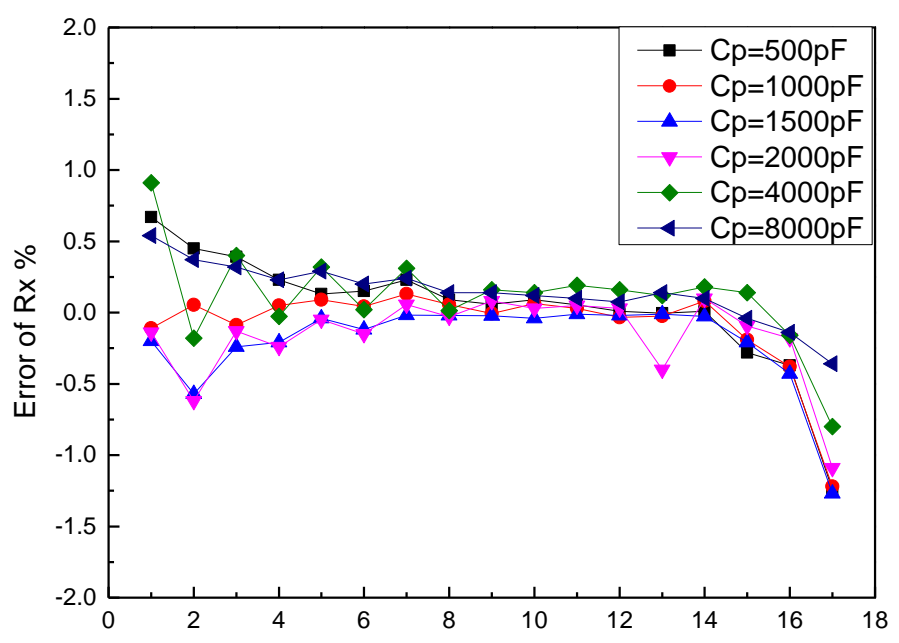

\section{Figure 6. Relative Errors of Resistance with Different Distributed Capacitance}

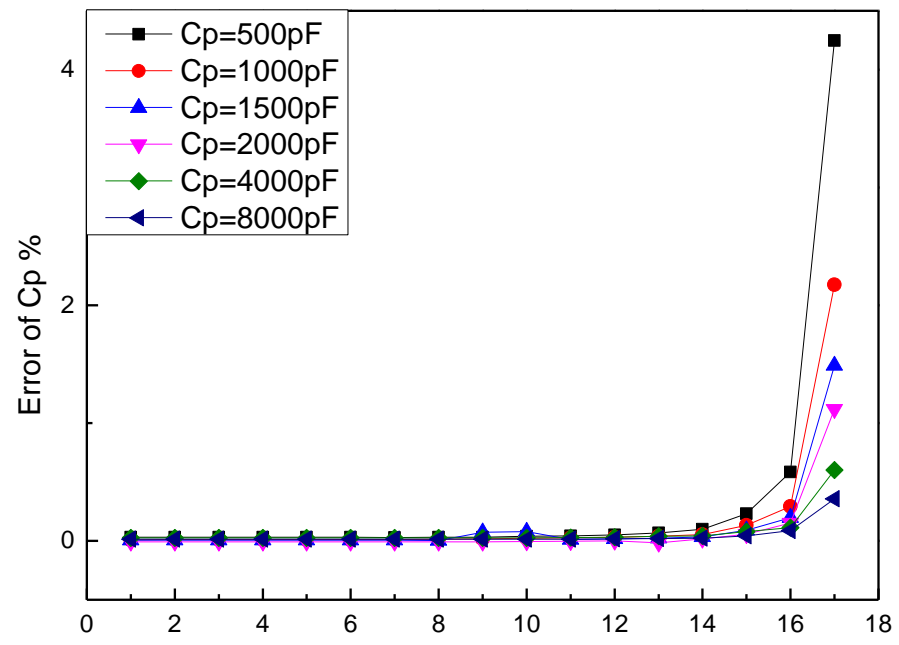

Figure 7. Relative Errors of Calculated Capacitance

\subsection{Verification of Irrelevance to Excitation Source Amplitude}

The irrelevance of the measurement method to excitation source amplitude is verified by setting measuring resistance $R_{X}=81.842 \mathrm{~K} \Omega$, capacitance $C_{P}=2000 \mathrm{pF}, f_{1}=100 \mathrm{~Hz}$, $f_{2}=5600 \mathrm{~Hz}$, and changing the excitation source amplitude $E$. Switch $\mathrm{S} 1$ is switched to B, and the signal source which directly enters the blocking and buffer circuits without passing through the analog switch is taken as the excitation source. Switch S is switched to $\mathrm{B}$ to directly measure the amplitude of the excitation source (can be measured under $100 \mathrm{~Hz}$ conditions).

Because the signal source output passes through the blocking capacitor, the unipolar signal is converted into a bipolar signal, which reduces the signal amplitude by half during measurement. So the unipolar amplitude of signal source is measured value multiplied by 2 . Then, the switch is switched to A to measure the DC values under two frequencies, followed by iteration of time constant value and calculation of resistance value of measuring object. Signal source is changed to make the amplitude of square wave signal output changes between $0.5 \mathrm{~V} \sim 1.5 \mathrm{~V}$, a set of data is obtained as shown in Table 7. 
Table 8 shows 11 sets of data obtained by measurement under $R_{X}=81.842 \mathrm{~K} \Omega, C_{P}$ $=2000 \mathrm{pF}, f_{1}=100 \mathrm{~Hz}, f_{2}=5600 \mathrm{~Hz}$, and a variable amplitude of $E$ between $0.5 \sim 1.5 \mathrm{~V}$. At different excitation source amplitudes, the obtained time constant value $\tau$ remains essentially the same; the error line graph 2.12 shows that the error of measuring resistance $R_{X}$ is distributed within $1 \%$.

Table 7. Varies Resistance Verification Value Under $C_{p}=8000 p F$

\begin{tabular}{|r|r|r|r|r|r|r|}
\hline$E(\mathrm{~V})$ & $E_{1}(\mathrm{mV})$ & $v_{l}(\mathrm{mV})$ & $v_{12}(\mathrm{mV})$ & $\tau(\mu \mathrm{s})$ & $R_{X I}(\Omega)$ & $e(\%)$ \\
\hline 0.5 & 501.52 & 110.12 & 27.212 & 88.97 & 81.901 & 0.072 \\
\hline 0.6 & 602.18 & 132.21 & 32.681 & 88.939 & 81.886 & 0.054 \\
\hline 0.7 & 700.76 & 153.71 & 38.046 & 88.807 & 81.742 & -0.12 \\
\hline 0.8 & 800.1 & 175.81 & 43.491 & 88.865 & 81.84 & -0.0024 \\
\hline 0.9 & 900.06 & 197.51 & 48.851 & 88.881 & 81.808 & -0.042 \\
\hline 1 & 1001.88 & 219.75 & 54.381 & 88.828 & 81.736 & -0.13 \\
\hline 1.1 & 1099.52 & 241.1 & 59.686 & 88.792 & 81.694 & -0.18 \\
\hline 1.2 & 1199.4 & 263.07 & 65.083 & 88.855 & 81.735 & -0.13 \\
\hline 1.3 & 1300 & 285.23 & 70.636 & 88.756 & 81.781 & -0.075 \\
\hline 1.4 & 1401.42 & 307.54 & 76.084 & 88.856 & 81.813 & -0.035 \\
\hline 1.5 & 1500.18 & 328.56 & 81.845 & 89.026 & 81.525 & -0.39 \\
\hline
\end{tabular}

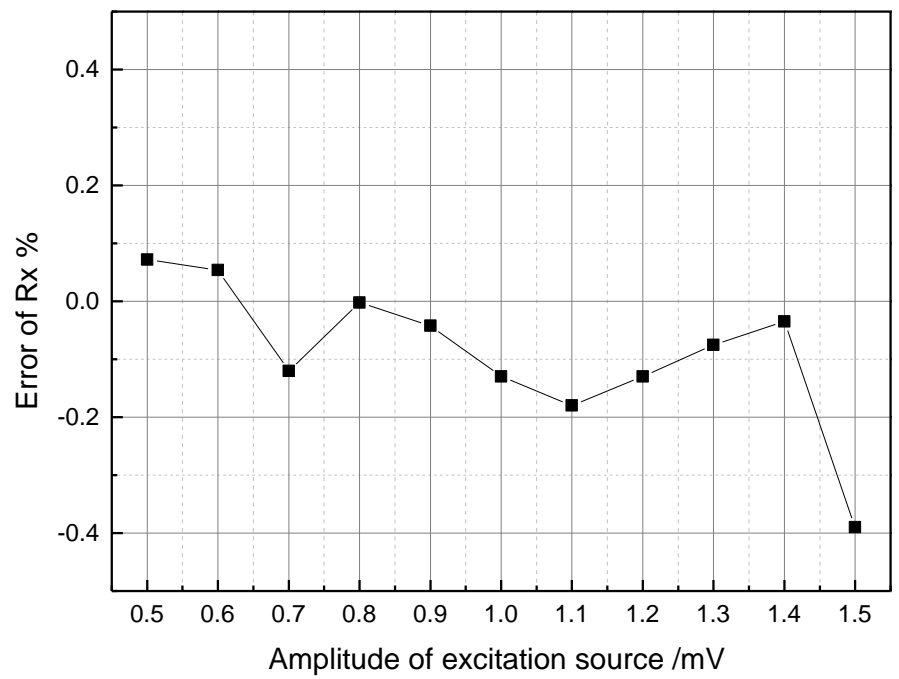

Figure 8. Measurement Errors of Resistance with Different Amplitude of Excitation Source

\section{Discuss}

As can be seen from Tables 1 6 and Figure 6 7 at different excitation frequencies, the method for decoupled measurement of resistance and capacitance through the amplitudefrequency characteristic of output signal has the relative error of measured resistance $R_{X}$ basically distributed within $1 \%$ at a measured resistance $R_{X}$ range of $1 \mathrm{~K}-1 \mathrm{M} \Omega$, and a measured capacitance $C_{P}$ range of $0 \sim 8000 \mathrm{pF}$, which indicates the feasibility of the method. Analyzing data in the tables, as the measuring resistance is measured through ordinary bridge circuit, its divider resistance $R_{1}=100 \mathrm{~K} \Omega$, due to the nonlinearity of bridge circuit, sensitivity is reduced when the measuring resistance is away from the divider resistance. So there is a relatively big error when the measuring resistance is very large (near $1 \mathrm{M} \Omega$ ) and very small (near $1 \mathrm{~K} \Omega$ ). To address it, the value of divider resistance $R_{1}$ 
can be changed accroding to the range of measuring resistance $R_{X}$, that is, increase the value of divider resistance at a larger resistance, and decrease the the value of divider resistance at a smaller resistance. Such change can be achieved through automatic range switching.

As can be seen from the Tables 2.1 2.6, when $R_{X}$ is within a $10 \mathrm{~K} \sim 1 \mathrm{M} \Omega$ range, the calculated distributed capacitance $C_{P 1}$ remains essentially the same, approaching the nominal value, the value also reflects the actual value of distributed capacitance. However, the $C_{P 1}$ value calculated at a $R_{X}$ range of $1 \mathrm{~K} \sim 10 \mathrm{~K} \Omega$ deviate rather largely from the actual value, presenting errors. The relative error of measuring resistance $R_{X}$, on the other hand, is still substantially distributed within $1 \%$. To explain this phenomenon, equation (2.4) is derived to give:

$$
\frac{d v}{v}=\frac{\frac{e^{-T / \tau}}{1+e^{-T / \tau}}+\frac{\tau}{T} \ln \frac{1+e^{-T / \tau}}{2}}{\frac{1}{2}+\frac{\tau}{T} \ln \frac{1+e^{-T / \tau}}{2}} \frac{d \tau}{\tau}+\frac{R_{1}}{R_{1}+R_{X}} \frac{d R_{X}}{R_{X}}
$$

Because $T \gg>$, so $\mathrm{e}^{-T / \tau} \rightarrow 0$, simplifying the formula (2.11) yields:

$$
\begin{gathered}
\frac{d v}{v}=\frac{-0.69 \frac{\tau}{T}}{\frac{1}{2}-0.69 \frac{\tau}{T}} \frac{d \tau}{\tau}+\frac{R_{1}}{R_{1}+R_{X}} \frac{d R_{X}}{R_{X}} \\
\text { Set } \alpha=\frac{-0.69 \frac{\tau}{T}}{\frac{1}{2}-0.69 \frac{\tau}{T}} \text { and } \beta=\frac{R_{1}}{R_{1}+R_{X}} \text { then } \\
\frac{d v}{v}=\alpha \frac{d \tau}{\tau}+\beta \frac{d R_{X}}{R_{X}}
\end{gathered}
$$

Since $T \gg>\tau$, and $\alpha$ is very small, when $R_{X}<<R_{1}, \beta$ approaches 1 . The relative error coefficient $\beta$ of measuring resistance $R_{X}$ is much greater than the relative error coefficient $\alpha$ of time constant $\tau$. Therefore, the measured time constant $\tau$ has very large relative error, whereas the resistance $R_{X}$ has relatively small error.

Then, partially deriving the equation (2.1) gives:

$$
\frac{d \tau}{\tau}=\frac{d C_{P}}{C_{P}}+\frac{R_{1}}{R_{1}+R_{X}} \frac{d R_{X}}{R_{X}}
$$

Error propagation process is $\frac{d v}{v} \rightarrow \frac{d \tau}{\tau} \rightarrow \frac{d R_{X}}{R_{X}} \rightarrow \frac{d C_{P}}{C_{P}}$. It can be seen from equation (2.16) that when $R_{X}$ is relatively small, most of the errors are diffused to the distributed capacitance. Therefore, it is easy to improve the accuracy when measurement is done at a low measuring resistance $R_{X}$. When the relative error of time constant $\tau$ is constant, resistance $R_{X}$ increases, the influence of relative error of $R_{X}$ enhances, the influence of relative error of distributed capacitance $C_{P}$ weakens, measurement accuracy of capacitance $C_{P}$ is improved, and $R_{X}$ measurement accuracy is reduced. Therefore, to improve the measurement accuracy at large measuring resistances, the measurement accuracy and resolution of DC voltage $v$ should be improved. 


\section{Conclusion}

Through the above analysis and data validation, it can be concluded that the measurement method is a proportional measurement method, whose measurement accuracy has nothing to do with the amplitude of excitation source. This conclusion is very important for the implementation of measuring devices, where square wave frequency can be produced by microcontroller port as the excitation source without requiring complex steady amplitude circuit, thus can effectively reduce the circuit design difficulty. Control and change of excitation frequency by microcontroller software allows automatic scanning of optimal excitation frequency, thus can effectively improve $v_{1}$ and $v_{2}$ difference, and reduce the calculation errors. Nonlinear equation solving can be achieved in embedded devices by table look-up, which can effectively reduce the operation time, and improve the instrument efficiency.

\section{Acknowledgments}

The present research is supported by National Natural Science Foundation of China (Grant No. 61472062) and the China Postdoctoral Science Foundation funded project (2015M571306).

\section{References}

[1] C. K. Johnson, K. M. Eskridge and D. L. Corwin, "Apparent soil electrical conductivity: Applications for designing and evaluating field-scale experiments", Comp. Electron. Agric., vol. 46, (2005), pp. 181202.

[2] A. E. Hoetink, T. J. C. Faes, K. R.Visser and R. M. Heethaar, "On the flow dependency of the electrical conductivity of blood", IEEE Trans. Biomedical Engineering, vol. 51, no. 7, (2004), pp. 1251-1261.

[3] L. Alwis, T. Sun and K. T. V. Grattan, "Optical fibre-based sensor technology for humidity and moisture measurement: review of recent progress", Measurement, vol. 46, no. 10, (2013), pp. 40524074.

[4] J. Nyström and E. Dahlquist, "Methods for determination of moisture content in woodchips for power plants-a review", Fuel, vol. 83, no. 7-8, (2004), pp. 773-779.

[5] Z. Szczepanik, Z. Rucki and Z. Moron, "Finite-element analysis of the electric field distribution in conductance cell", IEEE Trans. On Instrumentation and Measurement, vol. 52, no. 5, (2003), pp. 16481653.

[6] C. Q. Zhong, H. L. Han and L. Y. Zhang, "Summary of conductivity measurement", in IEEE Proceedings of the 6th World Congress on Intelligent Control and Automation, vol. 1, (2006), pp. 51065110.

[7] R. G. Jones, "Measurements of the electrical conductivity of water, in Science, Measurement and Technology", IEE Proceedings, vol. 149, no. 6, (2002), pp. 320-322.

[8] E. Ferrara, L. Callegaro and F. Durbiano, "Optimal frequency range for the measurement of A. C. conductivity in aqueous solutions", in Proceedings of the 17th IEEE Instrumentation and Measurement Technology Conference, vol. 44, no. 2, (2000), pp. 775-779.

[9] S. C. Bera, R. Sarkar and N. Mandal, "Study of the Effect of Excitation Frequency on Electrode Polarization Impedance-Type Flow Transducer", IEEE Trans. Instrumentation and Measurement, vol. 59, no. 12, (2010), pp. 3289-3295.

[10] J. J. Cilliers, W. Xie, S. J. Neethling, E. W. Randall and A. J. Wilkinson, "Electrical resistance tomography using a bidirectional current pulse technique", Meas. Sci .Technol, vol. 12, no. 8, (2001), pp. 997-1001.

[11] J. M. Keller, "Bipolar-pulse conductivity detector for ion chromatography", Anal Chem, vol. 53, no. 2, (1981), pp. 344-345.

[12] H. B. Huang, H. F. Ji, Z. Y. Huang and H. Q. Li, "Design of electrical resistance tomography system based on bidirectional pulsed current technique", In proceedings of the Sixth International Conference on Electronic Measurement and Instruments, vol. 1-3, (2003), pp. 2300-2302.

[13] A. Rajendran and P. Neelmaegam, "Measurement of conductivity of liquids using AT89C55WD microcontroller", Measurement, vol. 41, no. 1, (2004), pp. 59-63.

[14] G. L. Jiang and Y. P. Tian, "Design of Lock- in Amplifier in Measuring of Water Mill - conduction", Computer Measurement \& Control, vol. 15, no. 6, (2007), pp. 786-789.

[15] W. X. Chen and B. C. Hong, "A singlechip computer controlled conductivity meter based on conductance-frequency transformation”, Analytical Instrumentation, vol. 4, (2002), pp. 11-14. 
[16] N. M. Birlea, S. I. Birlea and E. Culea, “The skin's electrical time constants”, in IFMBE proceedings, vol. 36, (2011), pp. 160-163.

[17] Y. Yamamoto, H. Isshiki and T. Nakamura, "Instantaneous measurement of electrical parameters in a palm during electrodermal activity", IEEE Trans. on Instrumentation and Measurement, vol. 45, no. 2, (1996), pp. 483-487.

[18] R. Clerman, R. Joglekar, R. P. Ouellette and P. N. Cheremisinoff, "Biotechnology and energy use", Electrotechnology, vol. 8, (1981). 
International Journal of Control and Automation

Vol. 10, No. 6 (2017) 